\title{
Research Paper \\ The Relation between the Life Meaningfulness and the Level of Perceived Social Support among Elderly People Living in Nursing Homes in Isfahan
}

\author{
Atefe Ahmadi ${ }^{1}$, ${ }^{*}$ Hossein Abedi Parija², Mojtaba Habibi ${ }^{1}$, Elahe Sadeq ${ }^{1}$, Sorour Sadat Maddahi ${ }^{3}$ \\ 1. Department of Counseling, School of Educational Sciences and Psychology, Shahid Beheshti University, Tehran, Iran. \\ 2. Family Research Institute, Shahid Beheshti University, Tehran, Iran. \\ 3- Department of Counseling, Faculty of Humanities and Social Sciences, Islamic Azad University, Science and Research Branch, Tehran, Iran.
}

Citation: Ahmadi A, Abdi Parija H, Habibi M, Sadeqi E. [The Relation between the Life Meaningfulness and the Level of Perceived Social Support among Elderly People Living in Nursing Homes in Isfahan (Persian)]. Iranian Journal of Ageing. 2016; 1(4):118-125. http://dx.doi.org/10.21859/sija-1101118

http://dx.doi.org/10.21859/sija-1101118

Received: 22 Oct. 2015 Accepted: 12 Jan. 2016

Key words:

Perceived social support, The meaning of life, Elderly, Sanatarium

\section{A B S T R A C T}

Objectives The present study aimed to examine the life meaningfulness among the elderly people living in nursing homes of Isfahan, Iran based on their perceived social support from 3 sources of family, friends, and significant others.

Methods \& Materials In this research, a total of 120 older people (68 men and 52 women) were selected from the nursing homes of Isfahan through convenience sampling method, and their data were analyzed using Zimet et al. perceived social support and the life meaningfulness questionnaires (1988).

Results The results of stepwise and multiple regression analyses showed that $28.2 \%$ of individual differences regarding the life meaningfulness in the elderly people were related to the personal differences in their perceived social support (from family, friends, and significant others), in which the family and other significant persons had the most prominent effect on explaining the changes and in predicting the meaningfulness of life among the elderly. This correlation was statistically significant $(P=0.001)$. Based on analysis of variance, the explained regression model is also significant and linear because the value of $\mathrm{F}$ test for determining the effect of independent variables on life meaningfulness of the older people equals 15.353 , which is significant $(P=0.001)$. The significance of regression coefficients shows that the family significantly predicts the life meaningfulness of the elderly people. This means that $19.8 \%$ of variance is common between family and life meaningfulness of older people. This figure increases to $27.1 \%$ by adding significant others to the equation.

Conclusion The results showed that the older people who receive stronger social support also have significantly stronger feelings of meaning in their lives. Today with regard to social changes and busy life of children and accordingly their low relation with old parents, it is anticipated that the low perceived support from the family of the older people (which is the most important predicting factor of life meaningfulness among other factors of perceived social support) will affect the meaningfulness of their lives. Considering what older people told in their interviews with regard to the role of the family on promoting the quality of life among them and reaching a joyful experience of life meaningfulness, it is expected that by increasing the quality and quantity of relationship of the family members with older people, their needed support could be provided. Considering the effectiveness of perceived social support of older people on their life meaningfulness, this study generally showed that a higher attention to components of perceived social support is felt among this vulnerable group of older people.

\section{* Corresponding Author:}

Hossein Abedi Parija, MSc.

Address: Department of Counseling, School of Educational Sciences \& Psychology, Shahid Beheshti University, Evin, Tehran, Iran.

Tel: +98 (911) 7845027

E-mail: abedi005@gmail.com 


\section{رابطه معنادارى زندكى و حمايت اجتماعى ادراكشده در بين سالمندان ساكن آسايشكاههاى شمر أصفهان}

عاطفه احمدى'، "حسين عابدىيريجا'، مجتبى حبيبى'، الهه صادقى'، سرور سادات مداحى'

1- كروه مشاوره، دانشكده علوم تربيتى و روانشناسى، دانشكاه شهيدبهشتى، تهران، ايران.

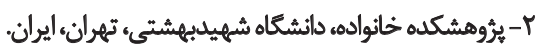

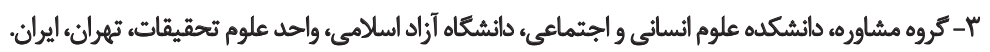

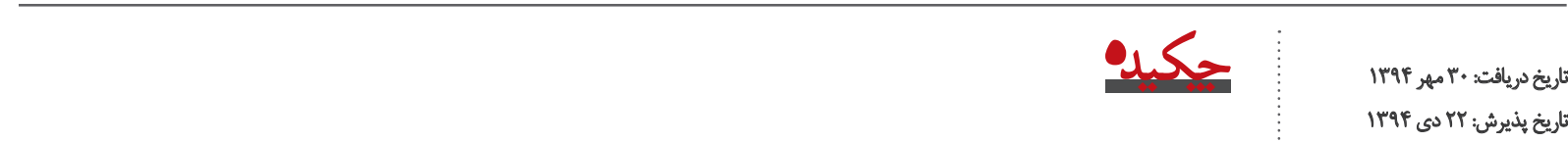

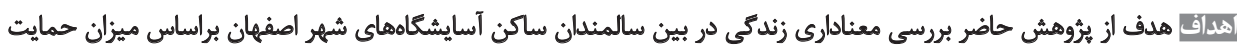

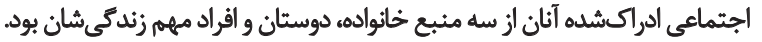

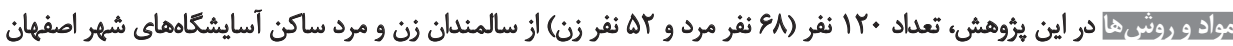

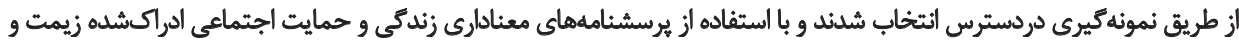

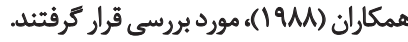

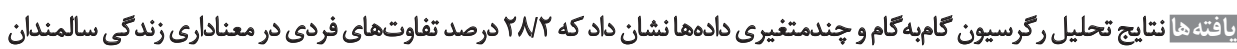

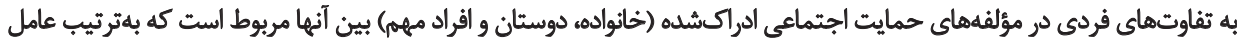

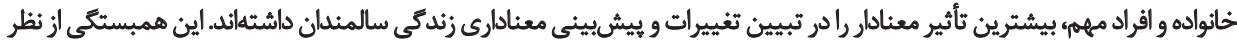

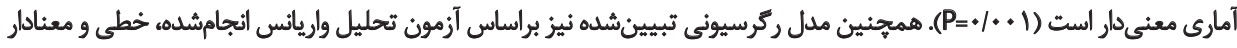

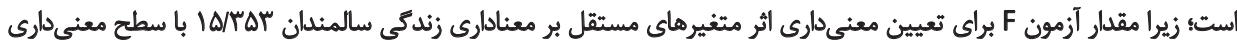

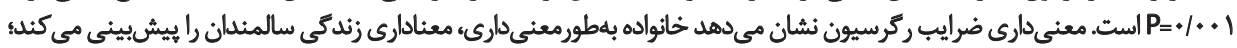

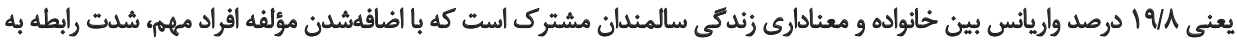

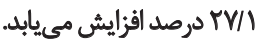

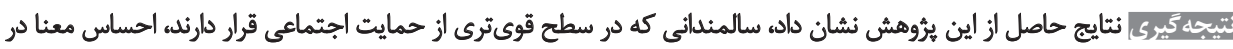

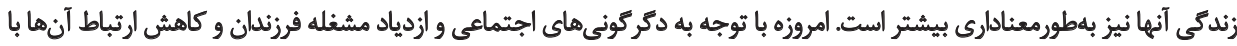

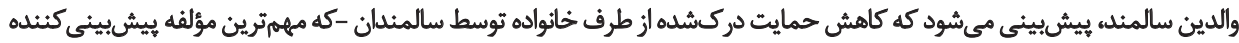

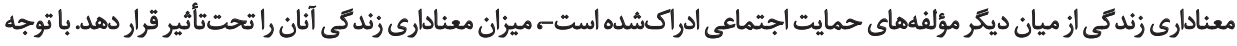

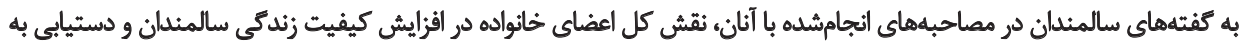

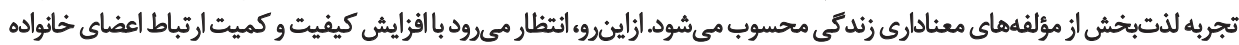

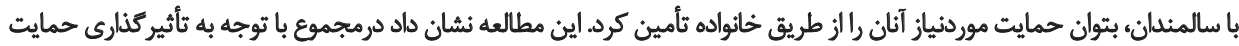

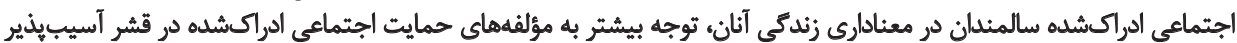

جمعيت اين كروه افزايش يافته است. براساس برآوردهاي انجامشده، مقام

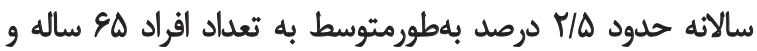

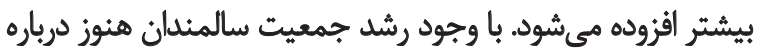

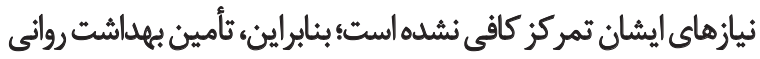

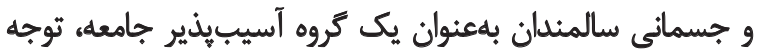

$$
\text { ويثراى رامىطلبد [1] }
$$

امروزه، سالمندى ' از يديدهاى مطرح سالهاى اخير در عرصه

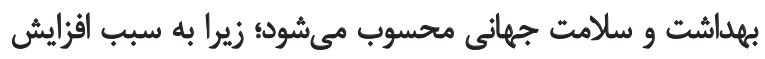

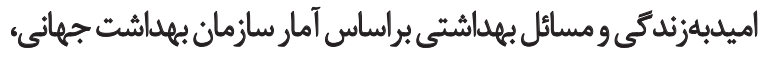

\section{Ageing}

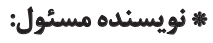

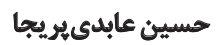

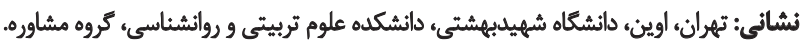
تلفن: يست الكترونيكي: قليف: abedi005@gmail.com 
حمايت اجتماعى ادراكشده" بر وضعيت جسمي، روائى، رضايت

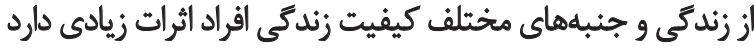

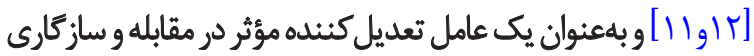

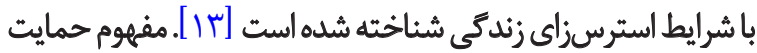

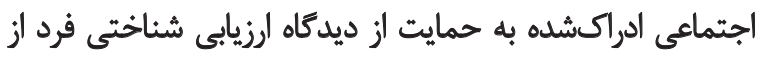

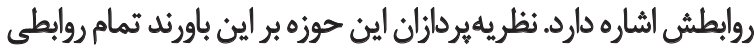

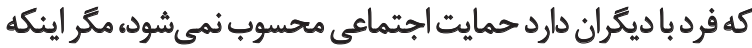

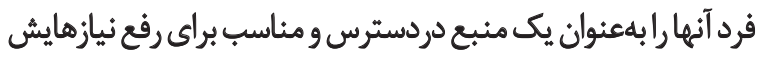

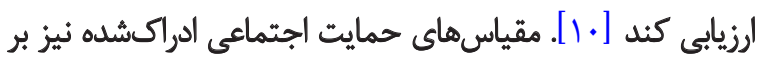

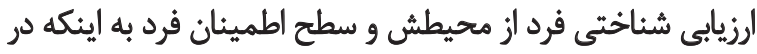

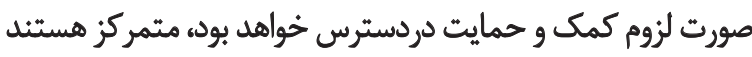

حمايتاجتماعى غير رسمى عامل مهمى در سالمئدى موفقيت آميز

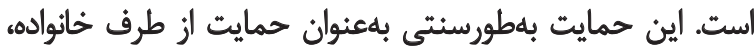

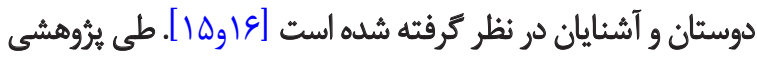

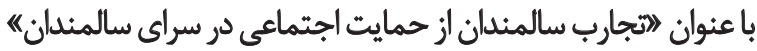

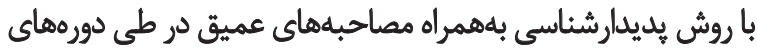

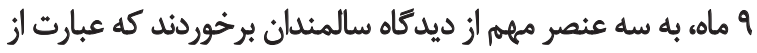

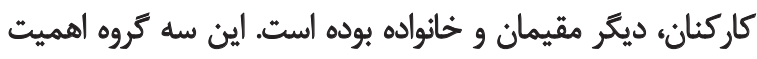

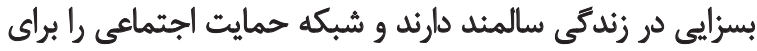

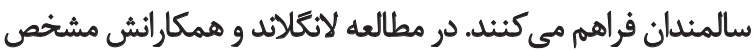

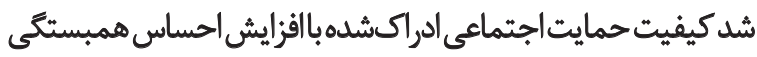

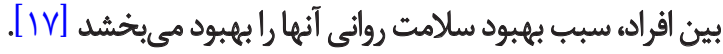

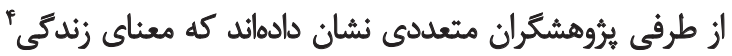

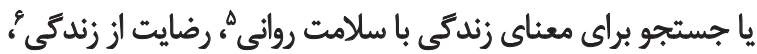

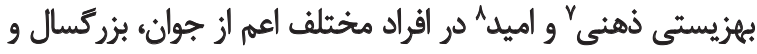

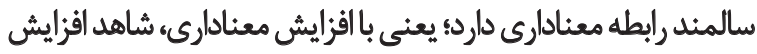
سلامت روانى و اميد و كاهش نشائهاى بيمارى و اختلالات اتلات روانى هستيم [1 [1 ].

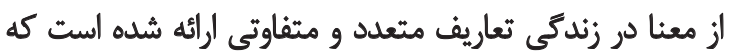

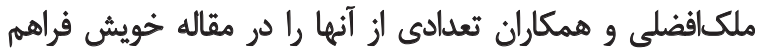

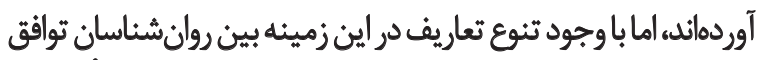

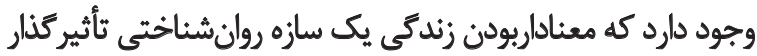

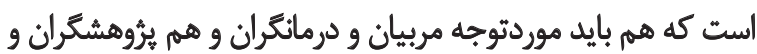

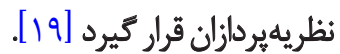

\footnotetext{
3. Perceived social support

4. Meaning of life

5. Mental health

6. Life satisfaction

7. Well-being

8. Hope
}

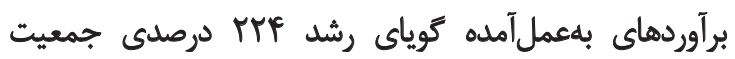

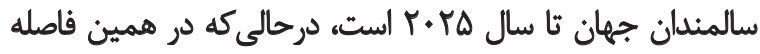

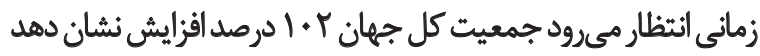

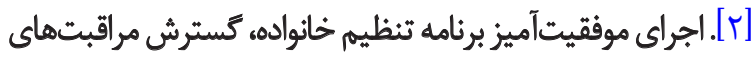

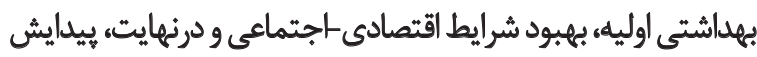

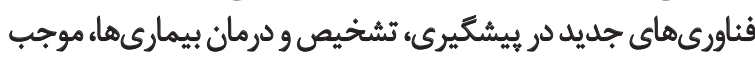

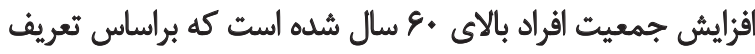

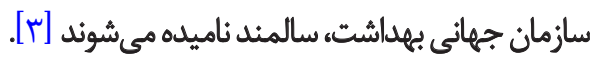

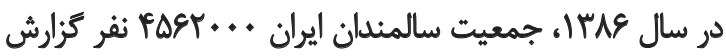

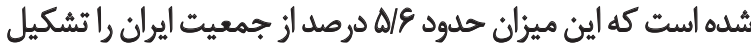

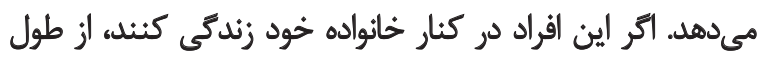

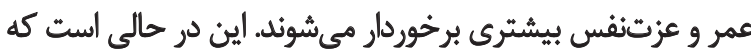

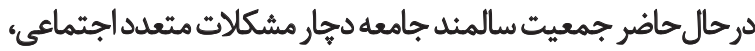

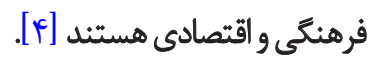

درصد ناثوانى سالمندان، با افزايش سن رشد مى يابد، بهطورى

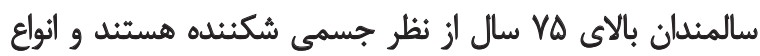

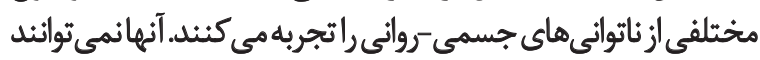

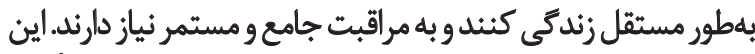
كروهاز سالمندان نيازهاى مراقبتى خود را راز طريق خافيت خانوادهها تأمين مي كنيد [ه].

باتوجه به تحقيقات كذشته، تفاوتهاي قابل توجهى بين تجربه إرائه

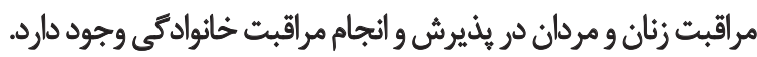

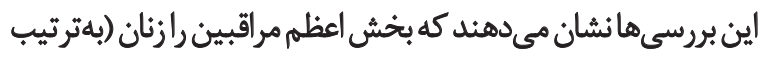

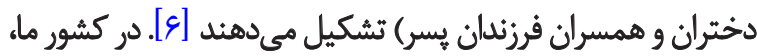

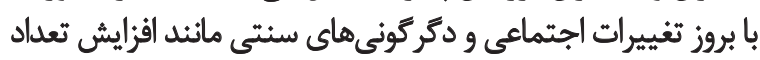

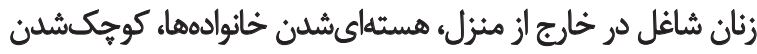

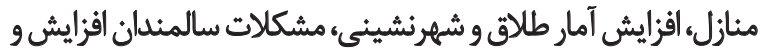

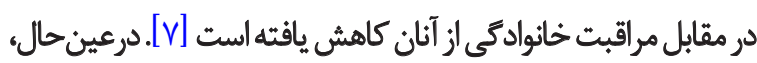

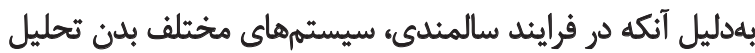

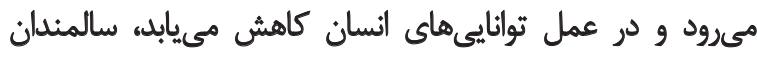
دجار افزايش ناثوانى، كاهش استقلال و افزايش وابستكى به به ديكران

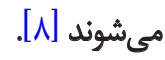

مطالعات نشان هي دهند كه سه عامل اساسى وضعيت سلامتى،

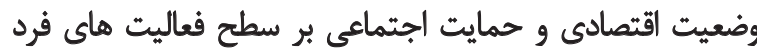

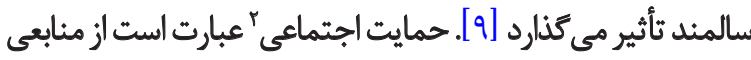

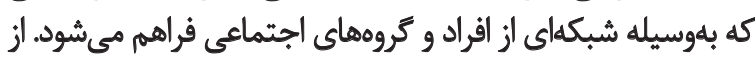

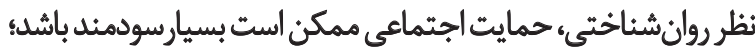

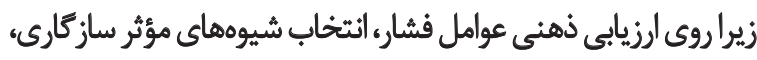

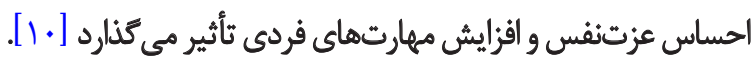




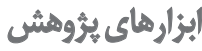

\section{ا. يرسشنامه احساس معنابخشبودن زندتى}

اين يرسشنامه توسط صالحى [Tr [ با استخراج از نظريات ويكتور

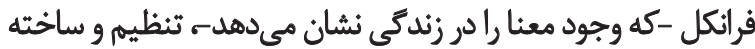

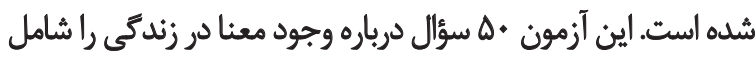

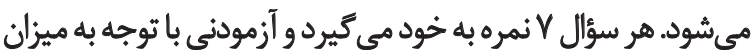

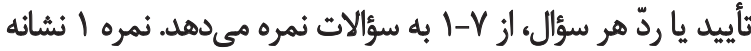

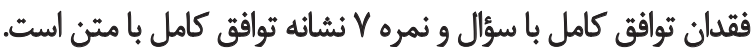

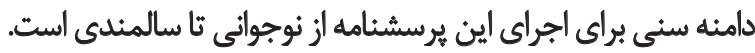

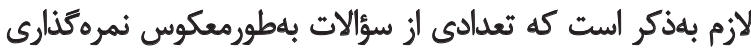

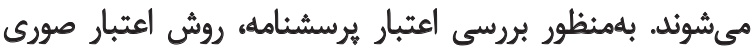

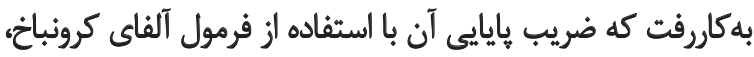

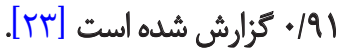

\section{T. يرسشنامه حمايت اجتماعى ادراكشده جُندبُعدى (MSPSS'9}

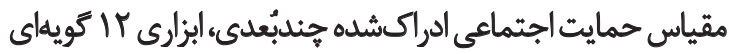

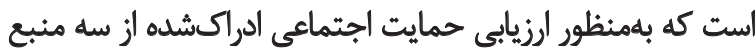

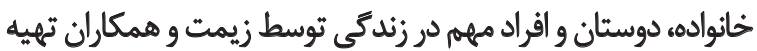

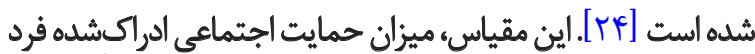

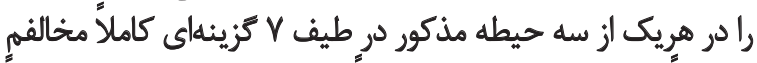

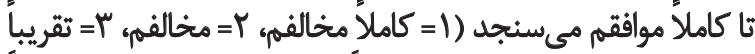

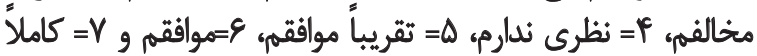

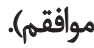

براى بلددست آوردن نمره كل اين مقياس، نمره همه كويهها با هم

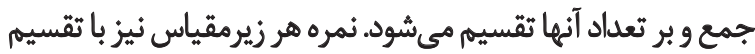

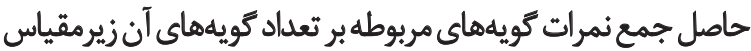

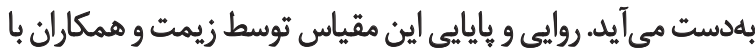

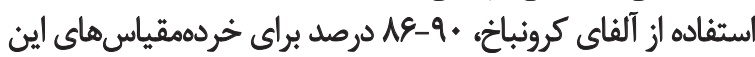

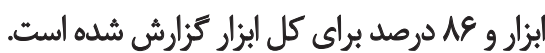

در ايران نيز اعتبار و روايى اين مقياس توسط سالمى و نيكيور

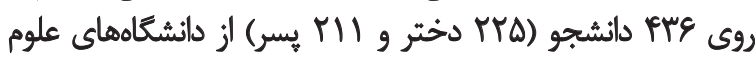

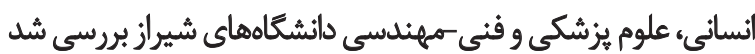

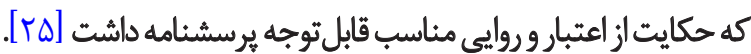

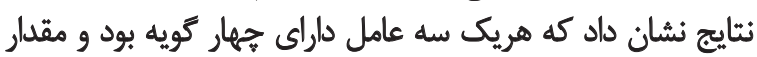

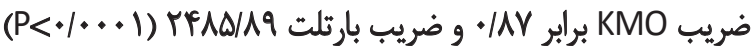

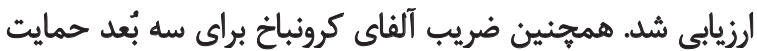

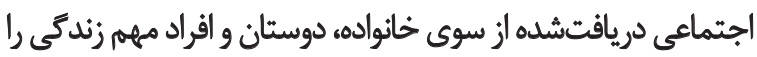

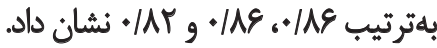

دادههاي جمعآورى شده با استفاده از نسخه 18 نرمافزار SPSS،

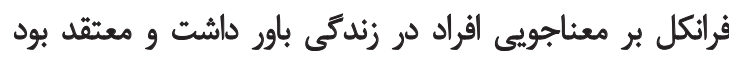

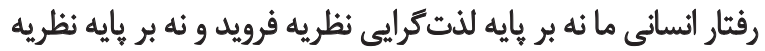

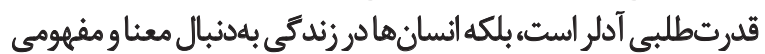

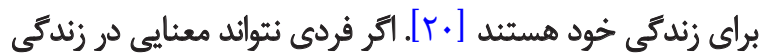

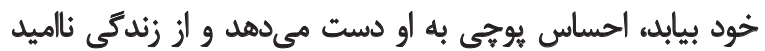

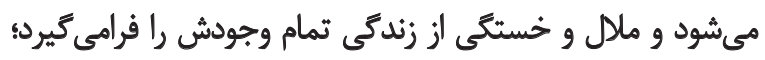

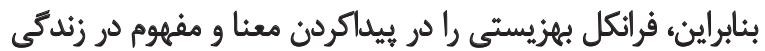

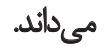

بهداشت روانى و اجتماعى از مهمترين مسائل جمعيت سالمثدان

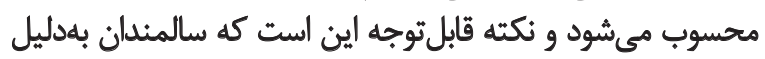

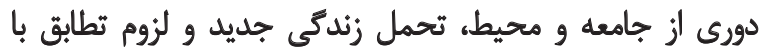

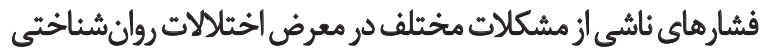

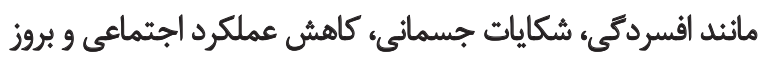

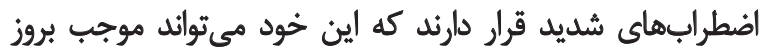

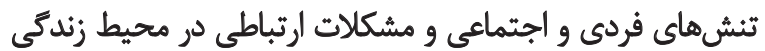

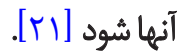

باتوجه به آنجهه درباره مشكلات سالمئدان ونيازهاى آنها كُقته شده.

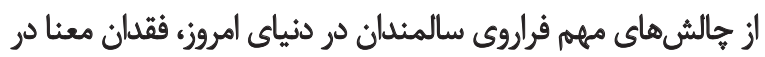

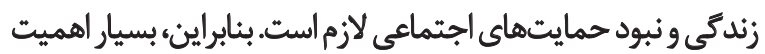

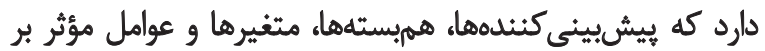

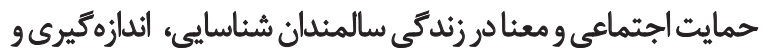

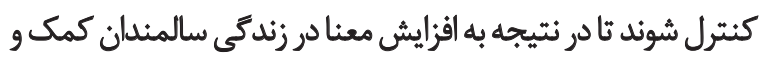
بر احساس مثبت آنها از زندكى افزوده شود.

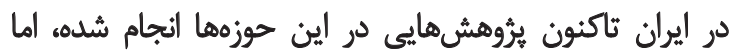

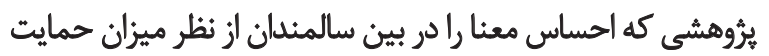

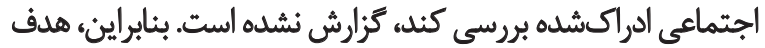

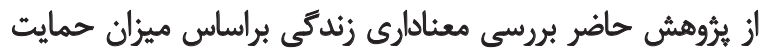

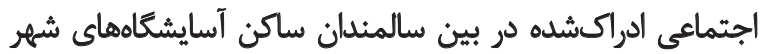

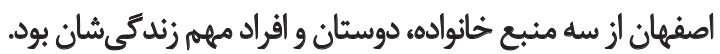

$$
\text { روش مطالعه }
$$

روش يُروهش حاضر از نوع توصيفى -همبستكى بود و جامعه آمارى

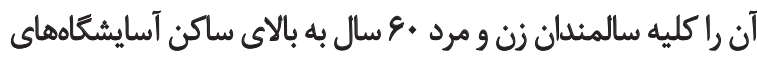

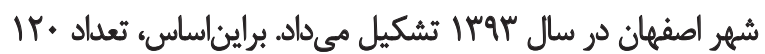

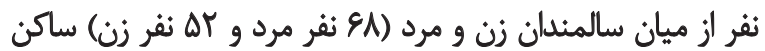

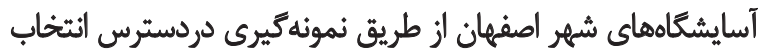

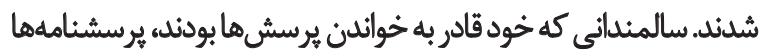

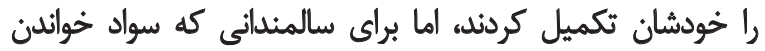

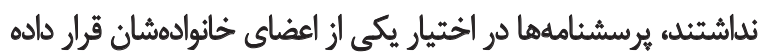

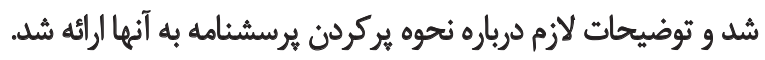

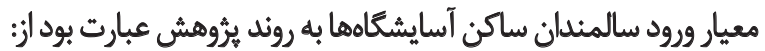

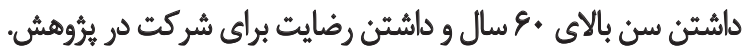


وجود دارد و همبستكى بين مؤلفههاى حمايت اجتماعى ادراكشده

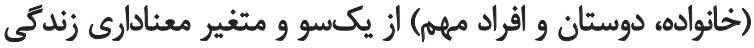

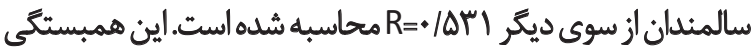

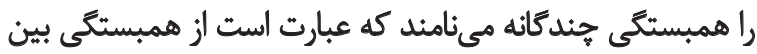

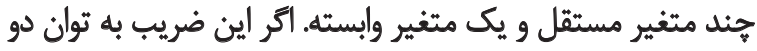

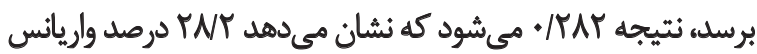

تحليل ركرسيون و ضريب همبستكى بيرسون مورد تجزيه و تحليل

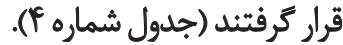

يافتهان

فرضيه يرُوهش حاضر بر آن بود كه بين معنادارى زندكى سالمندان

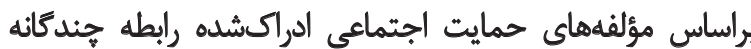
جدول ا. توزيع فراوانى و متغيرهاى جمعيت جشناختئ.

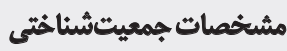

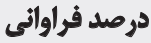

$\Delta$

pis

$9 / 8$

r.

YQ/AY

$\mathbb{W} / \Delta$

$V / \Delta$

$9 / 8$

rNMT

pq/

$\Delta / A T^{\prime}$

\section{فراواتى}

\&A

$\Delta r$

11

$\pi$

m

Pa

9

$\wedge$

is

$\Delta 9$

v
هرد

ij

بي سواد

ابتدايي

راهنمايي

ميزان تحصيلات

دييلم

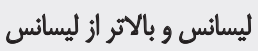

مجرد

مثتأهل

بيوه

مطلقه

\section{d}

جدول r. تحليل ركرسيون گَامبه كام (همبستكى جيندكانه).

\begin{tabular}{|c|c|c|c|}
\hline معنى دارى & خطاى استائدارد برأورد & مجذور همبستكى & همبستكى جئدكانه \\
\hline.$/ .+1^{* *}$ & $r r / q$ & - / TAr & $. / \Delta H^{\prime} \mid$ \\
\hline
\end{tabular}

L

جدول r. تحليل ركرسيون حندمتغيرى (كامبه كام).

\begin{tabular}{|c|c|c|c|c|c|c|c|}
\hline معني دارى & درجه آزادى Y & درجه آزادى 1 & $\mathrm{~F}$ & سهم خالص & مجذور ضريب رئرسيون & ضريب ركز سيون & مدل \\
\hline $.1 . .1 * *$ & 119 & 1 & rq/4T" &.$/ 194$ &.$/ 194$ &.$/ 470$ & خانواده \\
\hline $.1 . .1^{* *}$ & 111 & 1 & $11 / V \Delta \Delta$ &.$/ T \Delta q$ &.$/ \pi n$ & . $|\Delta T|$ & أفراد مهم \\
\hline
\end{tabular}

il

جدول F. ضرايب خام و استاندارد در تحليل ركرسيون جندمتغيرى.

\begin{tabular}{|c|c|c|c|c|c|}
\hline \multirow{2}{*}{ معنى داري } & \multirow{2}{*}{$\mathbf{T}$} & \multicolumn{2}{|l|}{ ضرايب استاثدارد } & ضرايب غيراستاندارد & \multirow{2}{*}{ مدل } \\
\hline & & بتا & خطاى انحراف معيار & B & \\
\hline $.1 \cdot .11^{* *}$ & IF/ATA & - & V/gT? & IFTMAF & ثابيت \\
\hline $.1 . .1^{* *}$ & F/MAT &.$/ m e q$ & $1 / 9.9$ & V/aAV & 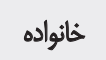 \\
\hline$+1 .+1^{* *}$ & r/erq & . MA & V/A9r & e/pqr & افراد مهم \\
\hline
\end{tabular}

次 


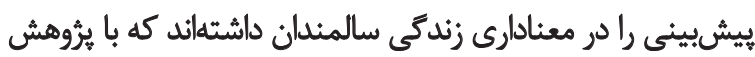

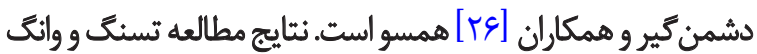

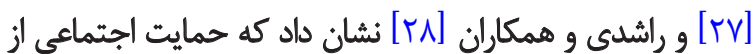

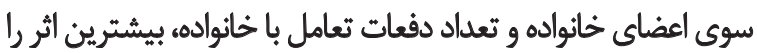

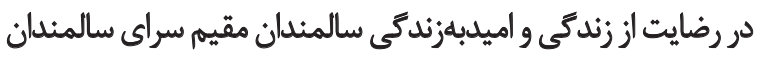

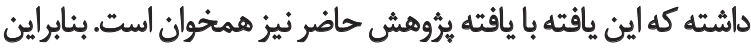

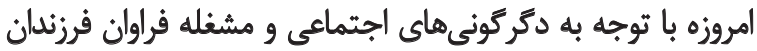

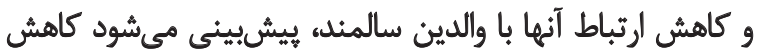

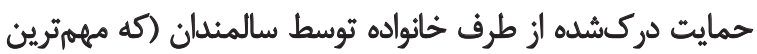

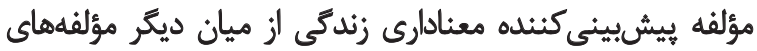

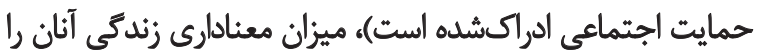
تحتثأثير قرار دهد.

براساس اظهارات سالمندان در مصاحبه هاى شان، نقش كل اعضاى خانواده در افزايش كيفيت زندكى سالمئدان و دستيابى به تجرئ

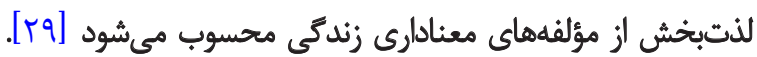

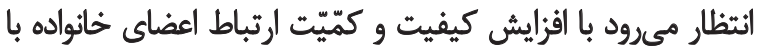

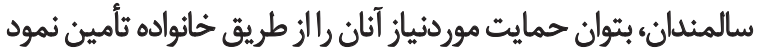

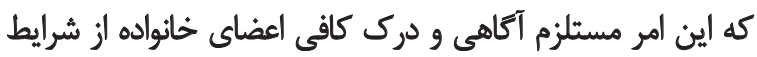
جسمانى و روانى سالمندان است.

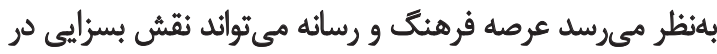

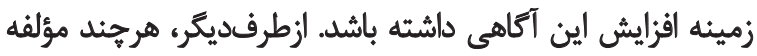

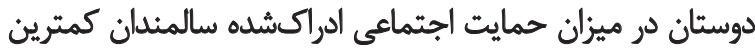

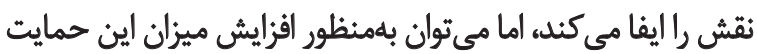

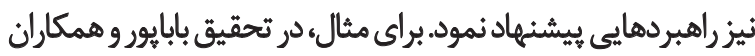

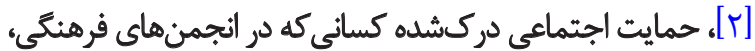

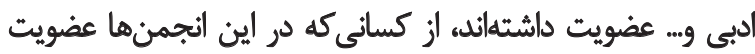

$$
\text { نداشتئد بيشتر بوده است. }
$$

از بين مؤلفه هاي حمايتاجتماعى، تنهاحمايت اجتماعى دركشده

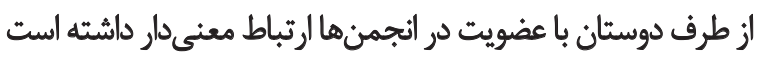

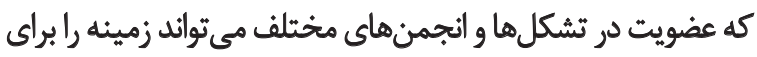

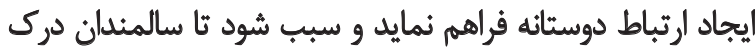

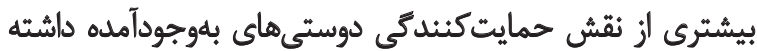

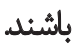

مطالعات كالاكر و تروكليو-لاندريكان [هائمان هم نشان داده است

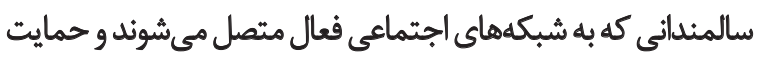

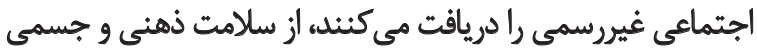

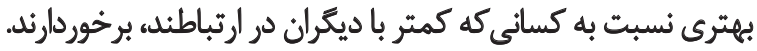

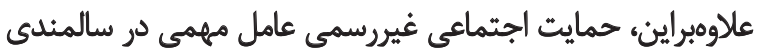

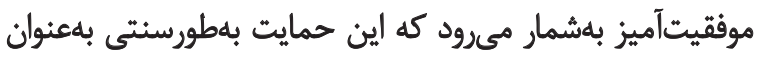
حمايت از طرف خانواده، دوستان و آشنايان در نظر ترفته شده است آنجر و همكاران معتقدند كه تأثيرات حمايت اجتماعى در دو جنس
يا تفاوتهاي فردى در معنادارى زندگى سالمندان مربوط به واريانس

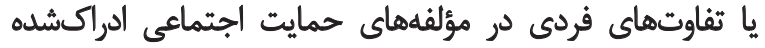
(خانواده، دوستان و افراد مهم) است.

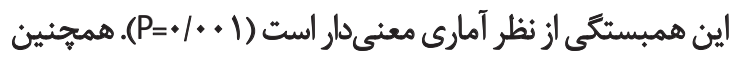

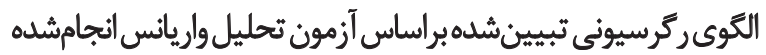

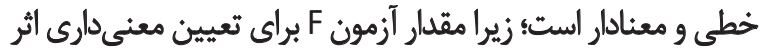

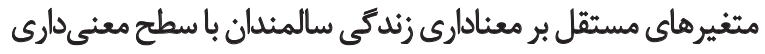

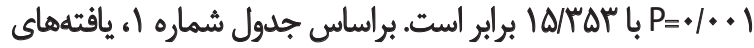

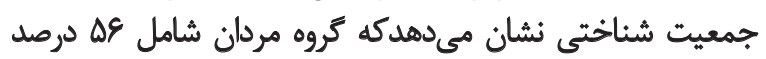

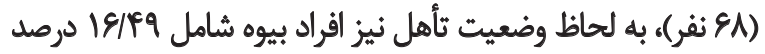

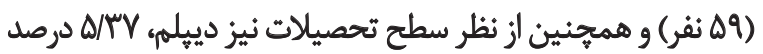

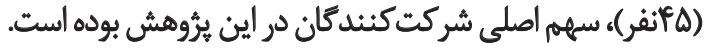
سؤال اصلى يثوهش حاضر اين بود كه اكداميك از مؤلفههاى

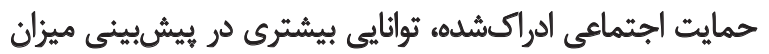

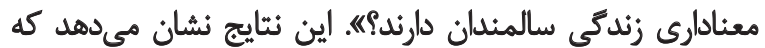

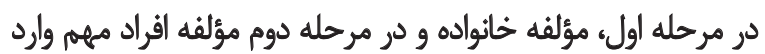

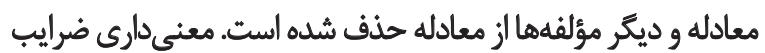

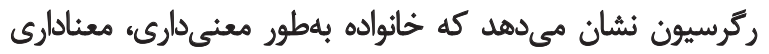

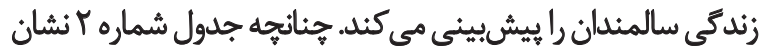

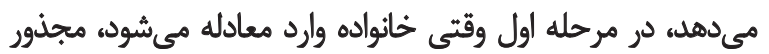

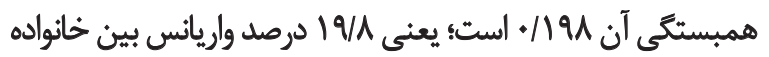

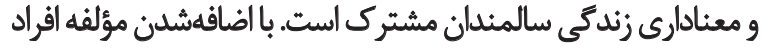

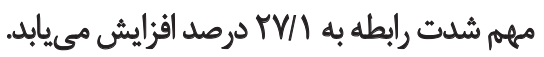
درمجموع، براساس اطلاعات جدول شماره بـ و با توجه به ضرايب

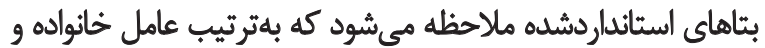

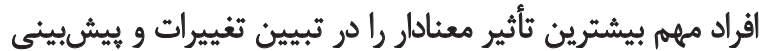
معنادارى زندكى سالمندان داشتهاند.

(أفراد مهي) IfFT/YAF

\section{ب0}

نتايج حاصل از اين يرُوهش نشان داد سالمندانى كه در سطح

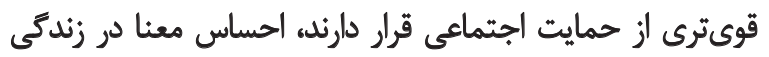

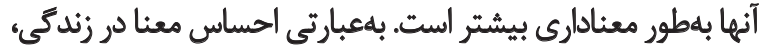

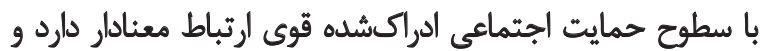

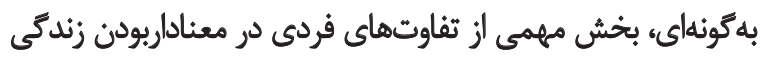

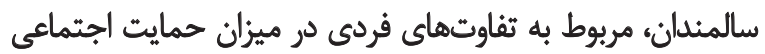

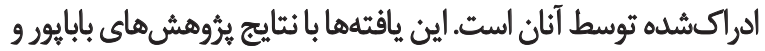

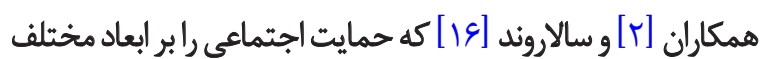

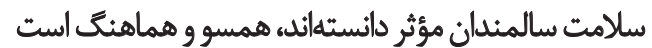
ديخًر يافته يُروهش حاضر اين بود كه از ميان سه مؤلفه خانواده،

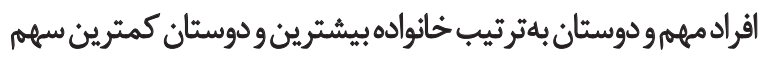




\section{References}

[1] Hemati Alamdarlo G, Ddhshiri GR, Shojaee S, Hakimirad E. [Health and loneliness status of the elderly living in nursing homes versus those living with their families (Persian)]. Iranian Journal of Ageing. 2009; 3(2):47-58.

[2] Babapour M, Raheb G, Eghlima M. [The Relationship between Social Support and Life Satisfaction among Elderly Nursing Home Residents in Tehran (Persian)]. Iranian Journal of Ageing. 2014; 9(1):12-16

[3] Khalili F, Sam S, Shariferad G, Hassanzadeh A, Kazemi M. [The relationship between perceived social support and social health of elderly of Esfahan city (Persian)]. Health Service Research. 2012; 7(6):12-15.

[4] Salarvand S, Abedi H, Hosseini H, Salehi S, Keyvanara M. [The emotional experiences of elderly people regarding the process of residency in nursing homes (Persian)]. Iran Journal of Nursing. 2007; 20(52):17-22

[5] Mohammadi F, Dabbaghi F, Yadavar Nikraveh M. [Facilitators factors and inhibiting at family care of frail elderly at home: Watchful women experience (Persian)]. Iranian Journal of Ageing. 2007; 2(4):445-453.

[6] Mohammadi F, Fallahi Khoshknab M, Khankeh HR. [Needs of caregivers to elderly patient with Alzheimer: Analysis the experience of family caregivers (Persian)]. Journal of Shahid Beheshti School of Nursing and Midwifery. 2009; 21(72):29-36.

[7] Delavar B. Examining the health status of elderly in the Islamic republic of Iran report. Paper presnted at: The $1^{\text {th }}$ International Conference of Aging in Iran; 1999 Oct. 19-21; Tehran, Iran.

[8] Melzer D, McWilliams B, Brayne C, Johnson T, Bond J. Profile of disability in elderly people: Estimates from a longitudinal population study. BMJ. 1999; 318(7191):1108-1111.

[9] Saberian M, Hajiaghajani S, Ghorbani R. [Survey of health, socialand economic conditions of the elderlyand their leisure time (Persian)]. Koomesh. 2004; 4(3):24-33.

[10] Lepore SJ, Evans GW, Schneider ML. Dynamic role of social support in the link between chronic stress and psychological distress. Journal of Personality and Social Psychology. 1991; 61(6):899-909.

[11] Clara IP, Cox BJ, Enns MW, Murray LT, Torgrudc LJ. Confirmatory factor analysis of the multidimensional scale of perceived social support in clinically distressed and student samples. Journal of Personality Assessment. 2003; 81(3):265-70

[12] Tajalli P, Sobhi A, Ganbaripanahah A. The relationship between daily hassles and social support on mental health of university students. Procedia-Social and Behavioral Sciences. 2010; 5:99-103.

[13] Friedlander LJ, Reid GJ, Cribbie R, Shupak N. Social support, self-esteem, and stress as predictors of adjustment to university among first-year undergraduates. Journal of College Student Development. 2007; 48(3):259-74.

[14] Gülaçtı F. The effect of perceived social support on subjective well-being. Procedia-Social and Behavioral Sciences, 2010; 2(2):3844-3849

[15] Gallagher LP, Truglio-Londrigan M. Community support older adults' perceptions. Clinical Nursing Research. 2004; 13(1):3-23.
متففاوت معنا هي يابد و تفاوتهاي جنسيتى در شبكهائ اجتماعى رئي

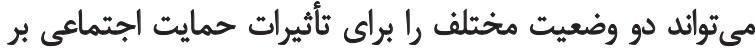

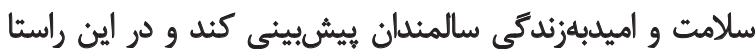

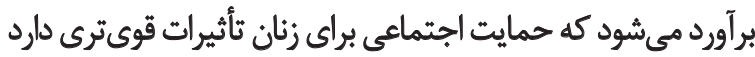

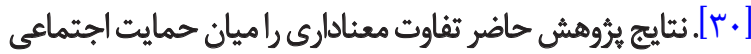

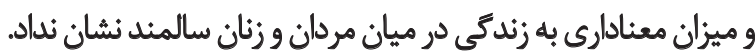

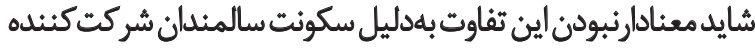
در اين يُوهش در آسايشكاه باشد. نتيجهيَيرىنهايى

اين مطالعه نشان داد درمجموع، با توجه به تأثير كذارى حمايت

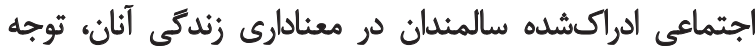

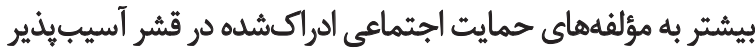

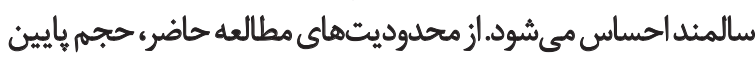

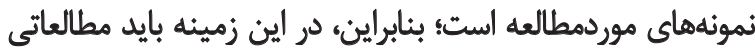

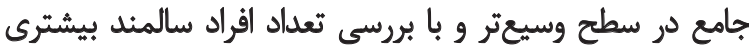

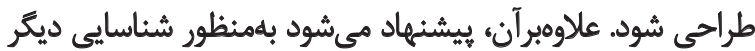

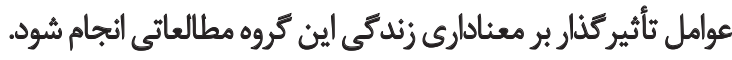
شركت در فعاليتهاى جمعى مى تواند كاركردهاى مثبث فراوانى

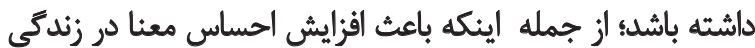

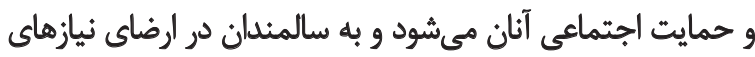

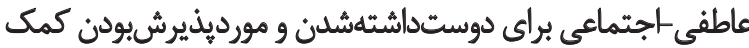

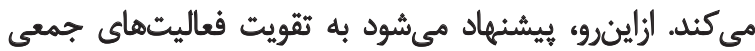

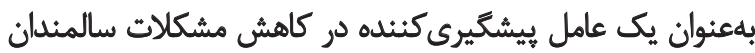

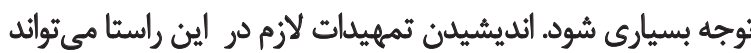
بر بهبود وضعيت روانشناختى و افزايش معنا در زندكى سالمندان

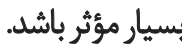

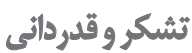

دريايان، از همكارى كاركنان آسايشعاهها و تمامى سالمنداني كه

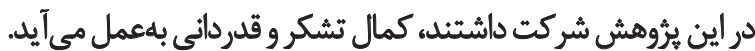


[16] Salarvand SH, Abedi H. [The Elders Experiences of Social Support in Nursing Home: A Qualitative Study (Persian)]. Iran Journal of Nursing. 2007; 20(52):39-50.

[17] Langeland E, Wahl AK. The impact of social support on mental health service users' sense of coherence: A longitudinal panel survey. International Journal of Nursing Studies. 2009; 46(6):830-7.

[18] Mascaro N, Rosen DH. Existential meaning's role in the enhancement of hope and prevention of depressive symptoms. Journal of Personality. 2005; 73(4):985-1014.

[19] Malekafzali H, Baradaran Eftekhari M, Hejazi F, Khojasteh T, Tabrizi R, Faridi T. [Community of mobilization for improve of elderly`s health in ekbatan district of Tehran (Persian)]. Hakim. 2005; 9(4):1-6.

[20] Fronkle V. Fonder of logotherapy overview on psychology and existencial psychotherapy [AR. Mohammadpour, Persian trans]. Tehran: Danjheh; 2005.

[21] Moghaddasi J, Mardani A, Nikfarjam M. [Efficacy of consoling guidelines on decreasing of psychological problems of newly arrived students of Shahrekord University of Medical Sciences (Persian)]. Shahrekord University of Medical Sciences Journal. 2005; 7(2):42-35.

[22] Salehi M. [Examining Iran`s adolescents and youth issues from the view of humanist psychologists (Persian)] [PhD thesis]. Tehran: Islamic Azad University; 1995.

[23] Jamali F. [The relationship between religious attitudes, signifying a feeling of life and mental health at the University of Tehran (Persian)] [MA thesis]. Tehran: University of Alzahra; 2003.

[24] Zimet GD, Dahlem NW, Zimet SG, Farley GK. The multidimensional scale of perceived social support. Journal of Personality Assessment. 1988; 52(1):30-41.

[25] Salami AR, Joukar B, Nikpour R. [Internet and communication: perceived social support and loneliness as antecedent variable (Persian)]. Psychological Studies. 2009; 5(3):81-102.

[26] Taghdisi MH, Doshmangir P, Dehdari T, Doshmangir L. [Influencing factors on healthy lifestyle from viewpoint of Elderly people: Qualitative study (Persian)]. Iranian Journal of Ageing. 2013; 7(4):47-58

[27] Tseng SZ, Wang RH. Quality of life and related factors among elderly nursing home residents in Southern Taiwan. Public Health Nursing. 2001; 18(5):304-11.

[28] Rashedi V, Rezaei M, Gharib M, Nabavi SH. [Social support for the elderly: Comparison between home and nursing home (Persian)]. Journal of North Khorasan University of Medical Sciences. 2013; 5(2):351-356.

[29] Rezvani A. [Comparison of two methods logotherapy and metacognitive instruction on reduce girls anxiety referrals to high schools centers (Persian)] [MA thesis]. Isfahan: University of Isfahan; 2009.

[30] Unger JB, McAvay G, Bruce ML, Berkman L, Seeman T. Variation in the impact of social network characteristics on physical functioning in elderly persons: MacArthur Studies of Successful Aging. Journals of Gerontology Series B: Psychological Sciences and Social Sciences. 1999; 54(5):245-51. 